OMNIVIS'02 Workshop on Omni-directional Vision, June, 2002, Copenhagen, Denmark (held with ECCV02)

\title{
Constant Resolution Omnidirectional Cameras
}

\author{
José Gaspar ${ }^{\dagger}$, Cláudia Deccó ${ }^{\ddagger}$, Jun Okamoto Jr ${ }^{\ddagger}$, José Santos-Victor ${ }^{\dagger}$ \\ $\dagger$ Instituto de Sistemas e Robótica, \\ Instituto Superior Técnico, \\ Av. Rovisco Pais, 1, \\ 1049-001 Lisboa - Portugal. \\ \{jag,jasv\}@isr.ist.utl.pt \\ ¥ Escola Politécnica da Univ. de São Paulo \\ Dep. de Engenharia Mecânica, \\ Av. Prof. Mello Moraes, 2231 CEP \\ 05508-900 São Paulo - SP Brazil. \\ \{ccgdecco,jokamoto\}@usp.br
}

\begin{abstract}
In this paper we present a general methodology for designing mirrors of catadioptric omnidirectional sensors encompassing linear projection properties, the so called constant resolution cameras. The linearity is stated between $3 D$ distances (or angles) and pixel coordinates.

We include three practical cases of interest of linear constraints for both standard (cartesian pixel distribution) and log-polar cameras: constant vertical, horizontal and angular resolution.

Finally, the formulation is applied in designing a camera combining some of the presented practical cases. Resulting images show that the design was successful as the desired linear properties were obtained.
\end{abstract}

\section{Introduction}

Sensor design is an enabling key for developing, simplifying or improving several applications in Computer Vision. In the recent past some traditional problems like scene representation, surveillance or mobile robot navigation, were found to be conveniently approached using different sensors and led to a significant effort in research and development of omnidirectional vision systems, i.e. systems capable of seeing in all directions [1, 2, 3, 4, 6, 7, 10, 12, 13, 15, 17].

There are many types of omnidirectional vision systems, being mostly common the ones based on rotating cameras, fish-eye lens or mirrors. The work described in this paper is mainly concerned with the omnidirectional vision systems combining cameras with mirrors, normally referred as catadioptric systems in the optics domain, specifically in what concerns the mirror profile design.

The image formation model of a catadioptric omnidirectional camera is determined by the shape of the mirror. In some cases, one can design the shape of the mirror in such a way that certain world-to-image geometric properties are preserved - which we will refer to as linear projection properties.

The choice of the properties that should be preserved by the catadioptric imaging system is naturally related to the specific application at hand: e.g. tracking and visual navigation. Specific details on the applications based on these omnidirectional cameras can be found in $[4,6,16]$. The desired linear projection properties, can be categorised into three main types:

- Constant vertical resolution - This design constraint aims to produce images, where objects at a (prespecified) fixed distance from the camera's optical axis will always be the same size in the image, independent of its vertical coordinates. As a practical example this viewing geometry would allow for reading signs or text on the surfaces of objects with minimal distortion. As another example, tracking is facilitated by reducing the amount of distortion that an image target undergoes when an object is moving in 3D. Finally, in visual navigation it helps by providing a larger degree of invariance of image landmarks w.r.t the viewing geometry.

- Constant horizontal resolution - The constant horizontal resolution ensures that the ground plane is imaged under a scaled Euclidean transformation. As such, it greatly facilitates the measurement of distances and angles directly from the image as well as easier tracking of points lying on the pavement thus having a large impact on robot navigation algorithms.

- Constant angular resolution - Here it is desired to ensure uniform angular resolution as if the camera had 
a spherical geometry. This sensor has interesting properties e.g. for ego-motion estimation [14].

Some of these designs have been presented in the literature, $[2,3,7,10]$ with a different derivation for each case. In this paper, instead, we present a unified approach that encompasses all the previous designs and allows for new ones. The key idea is that of separating the equations for the reflection of light rays at the mirror surface and the mirror Shaping Function, that explicits the linear projection properties to meet.

In some applications, one may be interested in having one type of projection properties in a certain area of the visual field and other projection properties in other areas of the visual field. We present a so-called Combined Mirror where the outer part of the image sensor is used to obtain a constant vertical resolution image, while the inner part is devoted to yield a constant horizontal resolution image. In this case, both constraints on the mirror shape resulting from the two design goals are combined together in a single profile.

Our general mirror design methodology is firstly developed for standard (cartesian) image sensors. However, a different choice of the sensor pixel layout may bring additional geometric and computational benefits for the resulting image/processing. Due to the rotational symmetry of the omnidirectional images, a natural choice is to use an image sensor with a polar structure. In this work we use the SVAVISCA [11] log-polar sensor developed at DIST, University of Genova. As a result of this mirror sensor combination, panoramic images can be directly read out from the sensor with uniform angular resolution and without requiring any additional processing or image warping. Our general design methodology is applicable in exactly the same terms to this sensor layout.

This paper is organised as follows: Section 2 introduces the general approach for mirror profile design in the form of a differential equation based on a mirror shaping function. Section 3 shows shaping functions to set specific constant resolution properties on the sensor. Section 4 details the design of a combined mirror for a log-polar camera, instantiating some of the presented constant resolution properties. In Section 5 we analyse the resulting mirror. Images obtained with the designed sensor are shown. Finally, in Section 6, we present our conclusions and the future directions of our research.

\section{A General Differential Equation for Mirror Profile Design}

The image formation process is determined by the trajectory of rays that start from a 3D point, reflect in the mirror surface and finally intersect with the image plane. This pro- cess depends on the projection function that specifies a type of sensor.

Due to the rotational symmetry of the system we only need to consider the design of the mirror profile. The geometry of the image formation of the omnidirectional catadioptric camera is shown in Figure (1).

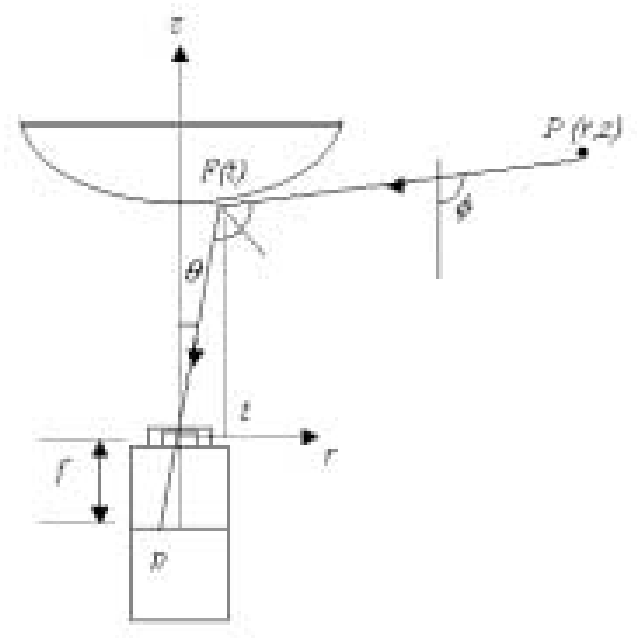

Figure 1. Main variables defining the projection equation.

Based on first order optics [9], and in particular on the reflection law, the following equation is obtained:

$$
\phi=\theta+2 . \operatorname{atan}\left(F^{\prime}\right)
$$

where $\theta$ is the camera vertical view angle, $\phi$ is the system vertical view angle, $F$ denotes the mirror shape (it is a function of the radial coordinate, $t$ ) and $F^{\prime}$ represents the slope of the mirror shape.

Equation (1) is valid both for single [1, 7, 13, 15, 17], and non-single projection centre systems $[2,3,4,6,10]$. When the mirror shape is known, it provides the projection function. For example, considering the single projection centre system combining a parabolic mirror, $F(t)=t^{2} / 2 h$ with an orthographic camera [13], one obtains the projection equation, $\phi=2 \operatorname{atan}(t / h)$ relating the (angle to the) 3D point, $\phi$ and an image point, $t$. Conversely, Eq.(1) can also be used to compute the mirror shape to achieve a desired projection function.

To simplify the addition of some desired projection properties (constraints) into Eq.(1) it is advantageous to replace the angular variables by cartesian coordinates. This is done assuming the pin-hole camera model and calculating the 
slope of the light ray hitting the mirror:

$$
\theta=\operatorname{atan}\left(\frac{t}{F}\right), \quad \phi=\operatorname{atan}\left(-\frac{r-t}{z-F}\right) .
$$

Then, expanding the trigonometric functions, one obtains a differential equation on the variables $F, t$ :

$$
\frac{\frac{t}{F}+2 \frac{F^{\prime}}{1-F^{\prime 2}}}{1-2 \frac{t F^{\prime}}{F\left(1-F^{\prime 2}\right)}}=-\frac{r-t}{z-F}
$$

where $(r, z)$ is a generic 3D point. This is Hicks and Bajcsy's differential equation relating 3D points to image points, $(t / F, 1)$ [10]. We assume without loss of generality that the focal length, $f$ is 1 , since it is easy to account for a different (desired) value at a later stage.

In order to numerically compute the solution of the differential equation, it is convenient to have the equation as an explicit expression for $F^{\prime 1}$.

Re-arranging Eq.(2) results in the following second order polynomial equation:

$$
F^{\prime 2}+2 \alpha F^{\prime}-1=0
$$

where $\alpha$ is a function of the mirror shape, $(t, F)$ and of an arbitrary 3D point, $(r, z)$ :

$$
\alpha=\frac{-(z-F) F+(r-t) t}{(z-F) t+(r-t) F} .
$$

We call $\alpha$ the mirror Shaping Function, since it ultimately determines the mirror shape by expressing the relationship that should be observed between 3D coordinates, $(r, z)$ and those on the image plane, determined by $t / F$. In the next section we will show that the mirror shaping functions allow us to bring the desired linear projection properties into the design procedure.

Concluding, to obtain the mirror profile first we specify the shaping function, Eq.(4) and then solve Eq.(3), or simply integrate:

$$
F^{\prime}=-\alpha \pm \sqrt{\alpha^{2}+1}
$$

where we choose the + in order to have positive slopes in the mirror shape, $F$.

\section{Setting Constant Resolution Properties}

Our goal is to design a mirror profile to match the sensor's resolution in order to meet, in terms of desired image properties, the application constraints. As seen in the previous section, the shaping function defines the mirror profile, and here we show how to set it accordingly to the design goal.

\footnotetext{
${ }^{1}$ E.g. having an explicit formula for $F^{\prime}$ allows to use directly matlab's ode45 function
}

Overall we want some world distances, $D$, to be linearly mapped to (pixel) distances, $p$, measured in the image sensor:

$$
D=a_{0} \cdot p+b_{0}
$$

for some values of $a_{0}$ and $b_{0}$ which mainly determine the visual field.

When considering conventional cameras, pixel distances are obtained by scaling metric distances in the image plane, $\rho$. In addition, knowing that those distances relate to the slope $t / F$ of the ray of light intersecting the image plane as:

$$
\rho=f \cdot \frac{t}{F}
$$

the linear constraint may be conveniently rewritten in terms of the mirror shape as:

$$
D=a \cdot t / F+b
$$

Notice that the parameters $a$ and $b$ can easily be scaled to account for a desired focal length, thus justifying the choice $f=1$ previously done.

In the following sections, we will specify which 3D distances, $D(t / F)$, should be mapped linearly to pixel coordinates, in order to preserve different image invariants (e.g. ratios of distances or angles in certain directions).

\subsection{Constant Vertical Resolution}

The first design procedure aims at preserving relative vertical distances of points placed at a fixed distance, $C$, from the camera's optical axis. In other words, if we consider a cylinder of radius, $C$, around the camera optical axis, we want to ensure that ratios of distances, measured in the vertical direction along the surface of the cylinder, remain unchanged when measured in the image. Such invariance should be obtained by adequately designing the mirror profile - yielding a constant vertical resolution mirror.

The derivation described here follows closely that presented by Gaechter and Pajdla in [5]. The main differences consist of (i) a simpler setting for the equations describing the mirror profile and (ii) the analysis of numerical effects when computing the derivatives of the mirror-profile to build a quality index (section 5). We start by specialising the linear constraint in Eq.(8) to relate $3 \mathrm{D}$ points of a vertical line $l$ with pixel coordinates (see Fig.2):

$$
z=a \cdot t / F+b, r=C \text {. }
$$

Inserting this constraint into Eq.(4) we obtain the specialised shaping function:

$$
\alpha=\frac{-\left(a \frac{t}{F}+b-F\right) F+(C-t) t}{\left(a \frac{t}{F}+b-F\right) t+(C-t) F} .
$$




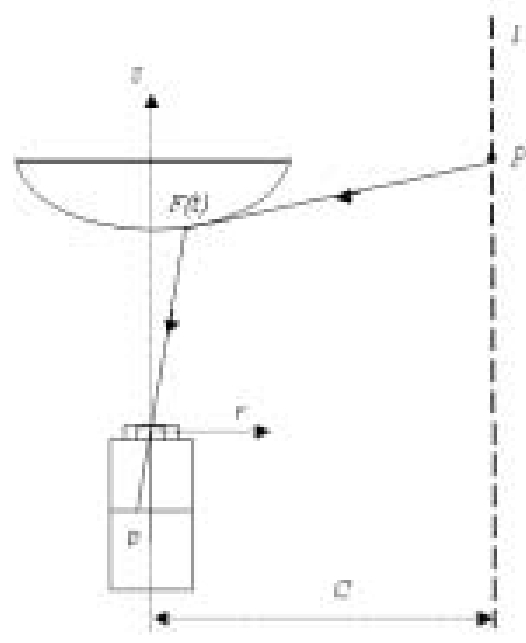

\section{Figure 2. Constant vertical resolution: points on the vertical line $l$ are linearly related to their projections in pixel coordinates, $p$.}

Hence, the procedure to determine the mirror profile consists of integrating Eq. (5) using the shaping function of Eq.(9), while $t$ varies from 0 to the mirror radius.

The initialization of the integration process is done by computing the value of $F(0)$ that would allow the mirror rim to occupy the entire field of view of the sensor.

\subsection{Constant Horizontal Resolution (Bird's Eye View)}

Another interesting design possibility for some applications is that of preserving ratios of distances measured on the ground plane. In such a case, one can directly use image measurements to obtain ratios of distances or angles on the pavement (which can greatly facilitate navigation problems or visual tracking). Such images are also termed Bird's eye views.

Figure (3) shows how the ground plane, $l$, is projected onto the image plane. The camera-to-ground distance is represented by $-C(C$ is negative because the ground plane is lower than the camera centre) and $r$ represents radial distances on the ground plane. The linear relation to image pixels is therefore expressed as:

$$
r=a \cdot t / F+b, z=C
$$

The linear constraint inserted into Eq.(4) yields a new shaping function:

$$
\alpha=\frac{-(C-F) F+\left(a \frac{t}{F}+b-t\right) t}{(C-F) t+\left(a \frac{t}{F}+b-t\right) F}
$$

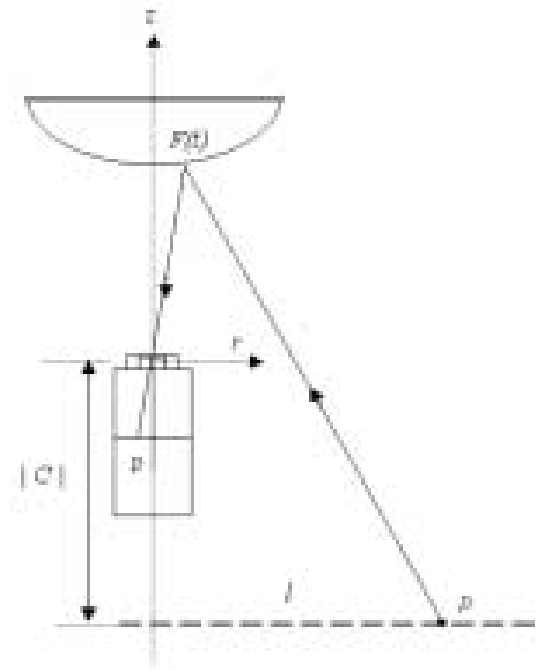

Figure 3. Constant horizontal resolution: points on the horizontal (radial) line $l$ are linearly related to their projections in pixel coordinates, $p$.

that after integrating Eq.(5) would result on the mirror profile proposed in [10].

\subsection{Constant Angular Resolution}

One last case of practical interest is that of obtaining a linear mapping from 3D points spaced by equal angles to equally distant image pixels, i.e. designing a constant angular resolution mirror.

Figure 4 shows how the spherical surface with radius $C$ surrounding the sensor is projected onto the image plane. In this case the desired linear property relates angles with image points:

$$
\varphi=a \cdot t / F+b
$$

and the spherical surface may be described in terms of $r, z$, the variables of interest in Eq.(4), simply as:

$$
r=C \cdot \cos (\varphi), z=C \cdot \sin (\varphi) .
$$

Then, replacing into Eq.(4) we finally obtain:

$\alpha=\frac{-\left(C \sin \left(a \frac{t}{F}+b\right)-F\right) F+\left(C \cos \left(a \frac{t}{F}+b\right)-t\right) t}{\left(C \sin \left(a \frac{t}{F}+b\right)-F\right) t+\left(C \cos \left(a \frac{t}{F}+b\right)-t\right) F}$.

Integrating Eq.(5), using the shaping function just obtained (Eq.(11)), would result in a mirror shape like the one of Chahl and Srinivasan [2] with the difference that in our case we are imposing the linear relationship from 3D vertical angles, $\varphi$ directly to image points, $(t / F, 1)$ instead of angles relative to the camera axis, $\operatorname{atan}(t / F)$. 


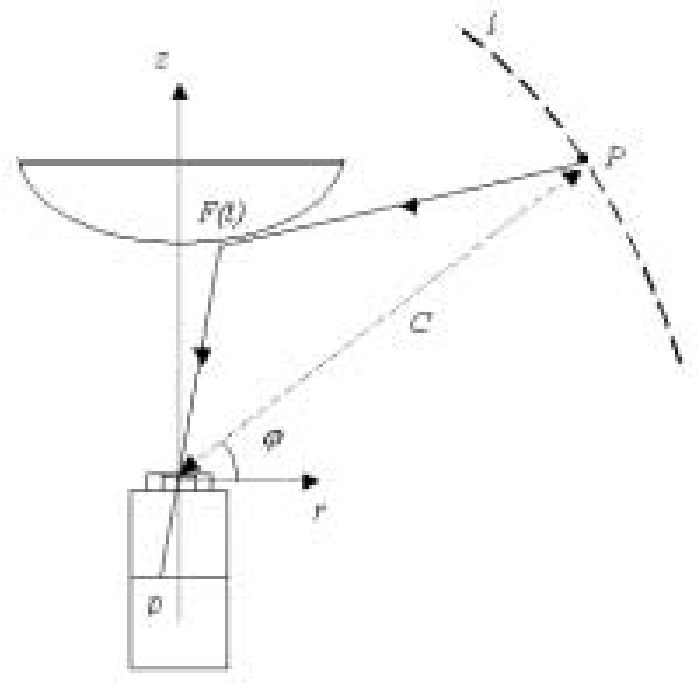

Figure 4. Constant angular resolution: points of the line $l$ on the surface of a sphere of radius $C$, are linearly related to their projections in pixel coordinates, $p$.

\subsection{Shaping functions for Log-polar Sensors}

Log-polar cameras differ from standard cameras both in density and organisation of the pixels. While a standard camera have equally sized pixels arranged in a matrix, a logpolar camera consists of a set of concentric circular rings, with a constant number of pixels, whose resolution decays logarithmically towards the image periphery. Hence, the relation of the linear distance, $\rho$, measured on the sensor's surface and the corresponding pixel coordinate, $p$, is specified by:

$$
p=\log _{k}\left(\rho / \rho_{0}\right)
$$

where $\rho_{0}$ and $k$ stand for the fovea radius and the rate of increase of pixel size towards the periphery.

As previously stated, our goal consists in setting a linear relation between world distances (or angles), $D$ and corresponding (pixel) distances, $p$ (see Eq.(6)). Combining into the linear relationship the pin-hole model, Eq.(7) and the logarithmic law defining pixel coordinates, Eq.(12) results in the following constraint:

$$
D=a \cdot \log (t / F)+b
$$

It is interesting to notice that the only difference in the form of the linear constraint between conventional and log-polar cameras, equations (8) and (13), is the replacement of the slope $t / F$ by its logarithm. Doing this same replacement directly in the shaping functions, results in the desired expressions for the log-polar camera.
Hence, when using a log-polar camera the shaping function becomes, in the case of constant vertical resolution:

$$
\alpha=\frac{-\left(a \cdot \log \frac{t}{F}+b-F\right) F+(C-t) t}{\left(a \cdot \log \frac{t}{F}+b-F\right) t+(C-t) F},
$$

in the case of constant horizontal resolution:

$$
\alpha=\frac{-(C-F) F+\left(a \cdot \log \frac{t}{F}+b-t\right) t}{(C-F) t+\left(a \cdot \log \frac{t}{F}+b-t\right) F},
$$

and finally, in the case of constant angular resolution:

$$
\begin{aligned}
\alpha= & \frac{-\left(C \sin \left(a \cdot \log \frac{t}{F}+b\right)-F\right) F}{\left(C \sin \left(a \cdot \log \frac{t}{F}+b\right)-F\right) t} \ldots \\
& \ldots \frac{+\left(C \cos \left(a \cdot \log \frac{t}{F}+b\right)-t\right) t}{+\left(C \cos \left(a \cdot \log \frac{t}{F}+b\right)-t\right) F} .
\end{aligned}
$$

As in the case of standard cameras, the mirror shape results from the integration of Eq.(5), only now using the shaping functions just derived.

As concluding comments on the presented mirrors, the constant vertical resolution is the same as in [5]. In the case of the constant angular resolution sensor, it would be an implementation of a spherical sensor giving a constant number of pixels per solid angle. This is similar to the case of Conroy and Moore [3] with the difference that due to the nature of the log-polar camera we do not need to compensate for lesser pixels when going closer to the camera axis.

\section{Combining Constant Resolution Properties}

In some applications, one may be interested in having different types of mappings for distinct areas of the visual field. This is typically the case for navigation where the constant vertical resolution mirror would facilitate tracking of vertical landmarks, while the Bird's eye view would make localization with respect to ground plane features easier.

For this reason, we have done a combined mirror design for the SVAVISCA log-polar camera (see Appendix A for details), where the central and inner parts of the sensor (fovea and inner part of the retina) are used for mapping the ground plane while the external part of the retina is used for representing the vertical structure. These three regions are represented respectively by $R_{1}, R_{2}$ and $R_{3}$ in Figure 5 and the corresponding mirror sections as $M_{1}, M_{2}$ and $M_{3}$.

As detailed in the appendix, the central part of the SVAVISCA camera, the fovea, has equally sized pixels while the external part, $R_{2}$ and $R_{3}$ in the figure, has an exponential growth of the pixel size in the radial direction.

In the process of computing the mirror profile each of the parts is obtained individually using the shaping function encompassing the desired constant resolution property and the local pixels distribution. Therefore, for $\left(R_{3}, M_{3}\right)$ 


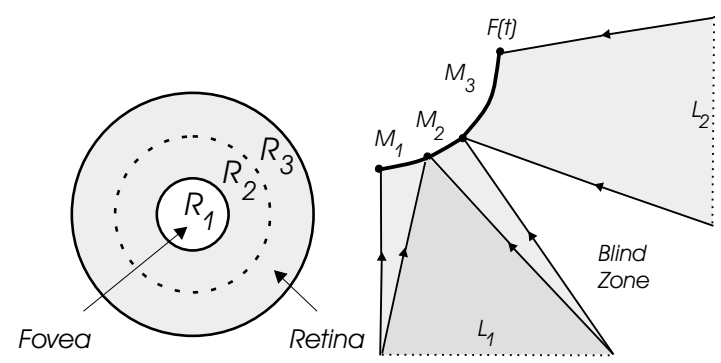

Figure 5. Combined mirror specifications. The horizontal line $L_{1}$ is reflected by the mirror sections $M_{1}$ and $M_{2}$, and observed by the sensor areas $R_{1}$ and $R_{2}$. The vertical line $L_{2}$ is reflected by $M_{3}$ and observed in $R_{3}$.

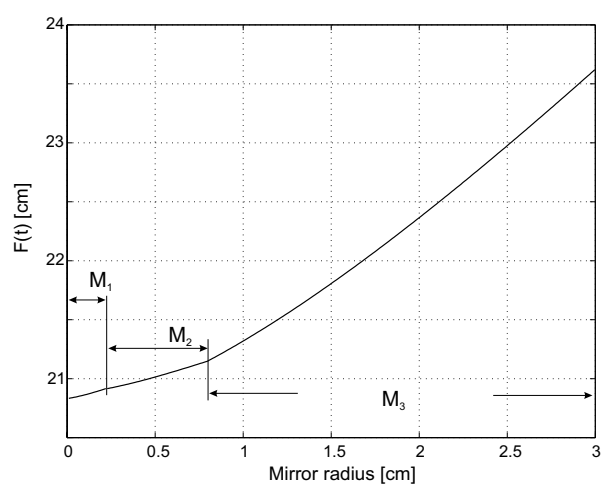

Figure 6. Combined mirror for the Svavisca log-polar image sensor. Computed profile.

we used the shaping function as given in Eq.(14) to impose constant vertical resolution in the case of a log-polar camera, while for $\left(R_{1}, M_{1}\right)$ and $\left(R_{2}, M_{2}\right)$ the expressions were respectively Eq.(10) and Eq.(15) to impose constant horizontal resolution for equally sized and exponentially growing pixels.

The field of view for each part of the sensor is determined by the corresponding parameters, $a$ and $b$, which determine the vertical/horizontal segments that must be mapped onto the image. Conversely minimal and maximal distances on the ground, heights on the vertical direction or angles to points on a sphere, determine the $a, b$ parameters.

Figures 6 and 7 show the obtained mirror comprising the three sections, the first two designed to observe the ground plane within distances $48 \mathrm{~cm}$ to $117 \mathrm{~cm}$ from the sensor axis, and the third one to observe $-10^{0}$ to $+25^{0}$ around the horizon line. The camera height was defined as $70 \mathrm{~cm}$, the radius of the cylinder as $200 \mathrm{~cm}$ and the focal length used was $25 \mathrm{~mm}$. The mirror radius was set to $3 \mathrm{~cm}$.

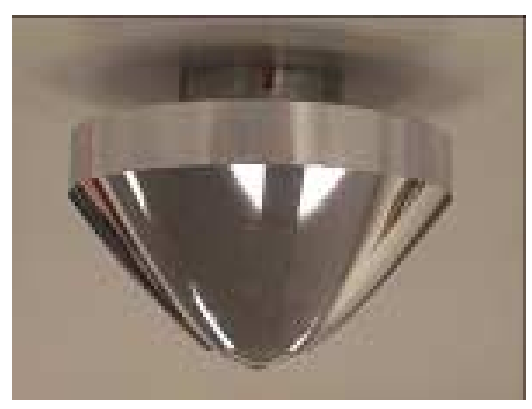

Figure 7. Manufactured mirror.

\section{Analysis of the Mirrors and Results}

The combined mirror described in the preceding section is composed by three parts. Here we analyze the quality of the outer part, designed to have constant vertical resolution. The other two parts would have similar analysis.

There are two main factors whose influence is important to discuss:

- Numerical Errors - As we do not have an analytic description of the mirror shape and the actual profile is obtained through numerical integration it is important to verify the influence of numerical integration errors in the overall process.

- Sensitivity - As the designed sensor does not have a single center of projection, the linear mappings obtained between pixel distances and world distances is only valid for specific world surfaces (e.g. specific vertical cylinders or horizontal planes in our case). How do the linear projection properties degrade for objects laying at distinct distances than those considered for the design?

As proposed in [5], the analysis of the mirror profile is done by calculating a quality index, $q(\rho)$. This quality index is defined as the ratio between the numerical estimate of the rate of variation of the $3 \mathrm{D}$ distances, $D$, with respect to distances in the image plane, $[\partial D / \partial \rho]_{n}$, and the corresponding theoretical value, $[\partial D / \partial \rho]_{t}$ :

$$
q(\rho)=\frac{[\partial D(\rho) / \partial \rho]_{n}}{[\partial D(\rho) / \partial \rho]_{t}}
$$

In the case under analysis, the theoretical value is obtained from Eq.(13) (noting that $t / F=\rho / f$ ) and the numerical value from the back-projection [8], which results directly from Eq.(2) given that the mirror shape is known.

For the perfect design process we should have $q(\rho)=$ 1. Computing $q(\rho)$ involves numerically differentiating the profile $F(t)$. Figure (8) shows some results obtained with different discrete approximations to derivatives. 


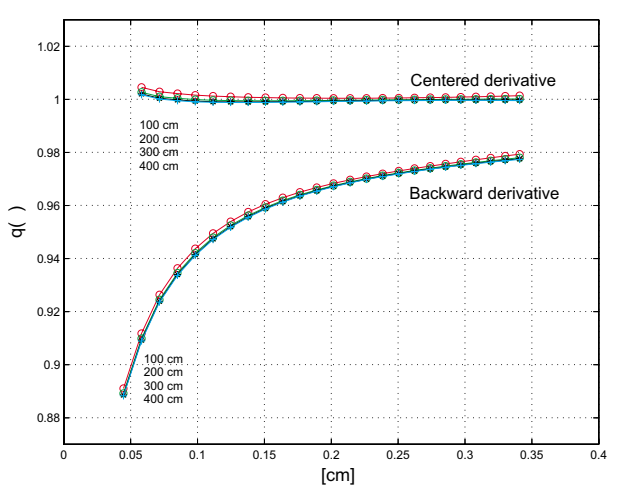

Figure 8. Analysis of the design criterion for different distances with a log-polar image sensor using different numeric approximations to the derivative: backward and centered differences.

These results show two main aspects. Firstly, the influence of varying distance with respect to the desired mapping properties does not seem to be too important, which suggests that we are close to the situation of a single projection center. Secondly, the way derivatives are computed is very important in terms of quality analysis. The simplest form of numerical differentiation leads to an error of about $10 \%$, while a better approximation shows that the computed profile meets the design specifications up to an error of about $1 \%$. Variations when the distance changes from the nominal $d=200 \mathrm{~cm}$ to $1 \mathrm{~m}$ or $4 \mathrm{~m}$ are not noticeable.

Figure 9 shows the combined mirror assembled with the camera and mounted on top of a mobile robot. The world scene contains vertical and ground patterns to test for the linear properties. Figure 10 shows an image as returned by the camera.
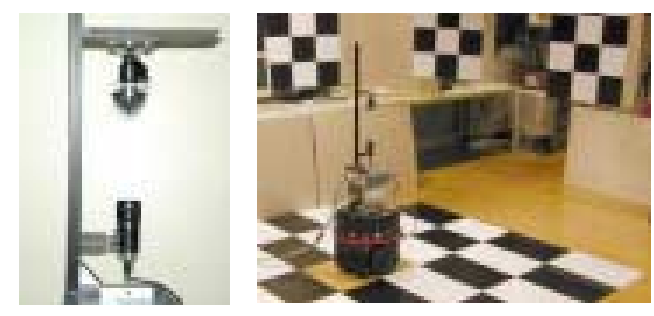

Figure 9. Svavisca camera equipped with the combined mirror (left) and world scene with regular patterns distributed vertically and over the floor (right).

Figure (11) shows resulting images. The panoramic im-

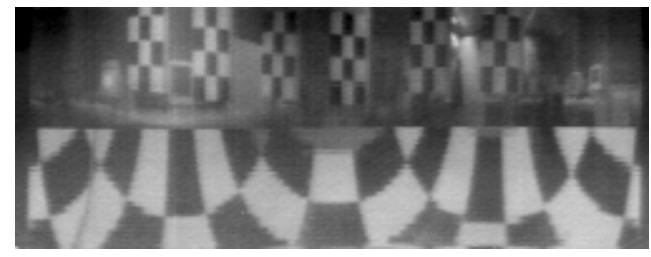

Figure 10. Image acquired with the Svavisca camera equipped with the Combined Mirror.

age results as a direct read out of the sensor (see Fig.11, top) and the bird's eye views are obtained after a change from cartesian to polar coordinates (Fig.11, bottom left and right). In the panoramic image the vertical sizes of black squares are equal to those of the white squares, thus showing linearity from 3D measures to image pixel coordinates. In the bird's eye views the rectilinear pattern of the ground was successfully recovered (the longer side is about twice the size of the shorter one).

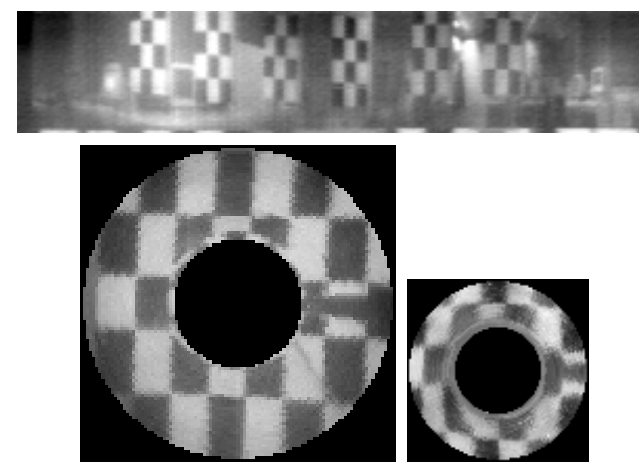

Figure 11. Images obtained from the original image in Fig.10: (top) panoramic and (bottom) bird's eye views. The bird's eye view at right originated from the fovea area.

\section{Conclusions}

In this paper we have described a general methodology to design and evaluate the profile of mirrors encompassing desired constant resolution properties. A function defining the mirror shape, the Shaping Function, was introduced and through it were presented formulas to achieve the following specifications:

- Constant vertical resolution mirror - distances measured in a vertical line at a fixed distance from the camera axis, are mapped linearly to the image, hence eliminating any geometric distortion. 
- Constant horizontal resolution mirror - where radial lines on the floor are mapped linearly in the image.

- Constant angular resolution mirror - equally spaced points on a meridian of a sphere are mapped linearly in the image plane.

The methodology considers also the case of log-polar cameras. The difference with the standard cameras case is an additional logarithmic relation that appears in the shaping function.

A prototype of a combined mirror has been built where the outer part of the sensor's retina is used for constant vertical resolution design; the inner part of the sensor's retina is used to generate the constant horizontal resolution criterion and the log-polar sensor's fovea has also been used, in spite of the reduced number of pixels, to generate a bird's eye view of the ground floor. Resulting images show that the design was successful as the desired linear properties were obtained.

In the future, we plan to use the new sensor for robot navigation and object tracking.

\section{Acknowledgements}

This work was partly funded by the European Union RTD - Future and Emerging Technologies Project Number: IST-1999-29017, Omniviews.

\section{A. SVAVISCA log-polar camera}

In this section we briefly revise the characteristics of the SVAVISCA log-polar sensor developed by DIST [11].

Inspired by the resolution of the human retina, the logpolar sensor is divided in two parts: the fovea and the retina. The fovea is the inner part of the sensor, with uniform pixel density and a radius of $\rho_{0}=0.273 \mathrm{~mm}$. Instead, the retina is the outer part of the sensor, consisting of a set of concentric circular rings, with a constant number of pixels, whose resolution decays logarithmically towards the image periphery. The sensor main specifications are summarized below:

- The fovea has 42 rings. The fovea has a constant pixelsize; the distribution of pixels is such that we have 1 pixel in the first ring, 6 in the second, an then 12, 18, 24 , etc, until reaching the number of 252 pixels in the $42^{\text {nd }}$ ring; The fovea radius is $\rho_{0}=272.73 \mu \mathrm{m}$;

- The retina has 110 rings, with 252 pixels each; The increase rate of pixel in the retina is $k=1.02337$;

- The total number of pixels is 33.193 with 5.166 in the fovea; The minimum size of pixel is $6.8 \times 6.45 \mu \mathrm{m}^{2}$; In the fovea the minimum pixel height is $6.52 \mu \mathrm{m}$; The diameter of sensor is $\rho_{\max }=7,135.44 \mu \mathrm{m}$.

\section{References}

[1] S. Baker and S. K. Nayar. A theory of catadioptric image formation. In IEEE ICCV'97, pages 35-42, January 1998.

[2] J. S. Chahl and M. V. Srinivasan. Reflective surfaces for panoramic imaging. Applied Optics (Optical Society of America), 36(31):8275-8285, November 1997.

[3] T. Conroy and J. Moore. Resolution invariant surfaces for panoramic vision systems. In IEEE ICCV'99, pages 392397, 1999.

[4] C. Deccó, J. Gaspar, N. Winters, and J. Santos-Victor. Omniviews mirror design and software tools. Technical report, Omniviews deliverable DI-3, available at http://www.isr.ist.utl.pt/labs/vislab/, September 2001.

[5] S. Gaechter, T. Pajdla, and B. Micusik. Mirror design for an omnidirectional camera with a space variant imager. In IEEE Workshop on Omnidirectional Vision Applied to Robotic Orientation and Nondestructive Testing, pages 99 105, August 2001.

[6] J. Gaspar, N. Winters, and J. Santos-Victor. Vision-based navigation and environmental representations with an omnidirectional camera. IEEE Transactions on Robotics and Automation, 16(6):890-898, December 2000.

[7] C. Geyer and K. Daniilidis. A unifying theory for central panoramic systems and practical applications. In ECCV'O0, pages 445-461 (vol2), June 2000.

[8] R. Hartley and A. Zisserman. Multiple View Geometry in Computer Vision. Cambridge University Press, 2000.

[9] E. Hecht and A. Zajac. Optics. Addison Wesley, 1974.

[10] A. Hicks and R. Bajcsy. Catadioptric sensors that approximate wide-angle perspective projections. In IEEE Workshop on Omnidirectional Vision - OMNIVIS'00, pages 97-103, June 2000

[11] LIRA-Lab. Document on specification. Technical report, Esprit Project n. 31951 - SVAVISCA - available at http://www.lira.dist.unige.it - SVAVISCA - GIOTTO Home Page, May 1999.

[12] F. Marchese and D. Sorrenti. Omni-directional vision with a multi-part mirror. In Fourth Int. Workshop on Robocup, pages 289-298, 2000.

[13] S. K. Nayar. Catadioptric omnidirectional camera. In IEEE CVPR'97, pages 482-488, Puerto Rico, June 1997.

[14] R. Nelson and J. Aloimonos. Finding motion parameters from spherical motion fields (or the advantage of having eyes in the back of your head). Biological Cybernetics, 58:261-273, 1988.

[15] T. Svoboda, T. Pajdla, and V. Hlavác. Epipolar geometry for panoramic cameras. In ECCV'98, pages 218-231, Freiburg Germany, July 1998.

[16] N. Winters, J. Gaspar, A. Bernardino, and J. SantosVictor. Vision algorithms for omniviews cameras. Technical report, Omniviews deliverable DI-3, available at http://www.isr.ist.utl.pt/labs/vislab/, September 2001.

[17] K. Yamazawa, Y. Yagi, and M. Yachida. Obstacle detection with omnidirectional image sensor hyperomni vision. In IEEE ICRA, pages 1062-1067, 1995. 